\title{
The case of equality in the Livingstone-Wagner Theorem
}

\author{
David Bundy $\cdot$ Sarah Hart
}

Received: 12 January 2007 / Accepted: 21 February 2008 / Published online: 4 March 2008

(C) Springer Science+Business Media, LLC 2008

\begin{abstract}
Let $G$ be a permutation group acting on a set $\Omega$ of size $n \in \mathbb{N}$ and let $1 \leq k<(n-1) / 2$. Livingstone and Wagner proved that the number of orbits of $G$ on $k$-subsets of $\Omega$ is less than or equal to the number of orbits on $(k+1)$-subsets. We investigate the cases when equality occurs.
\end{abstract}

Keywords Livingstone-Wagner Theorem · Permutation groups · Orbits · Partitions

\section{Introduction}

Throughout this article we let $G$ be a permutation group acting on a set $\Omega$ of size $n \in \mathbb{N}$ and let $1 \leq k<(n-1) / 2$. In [7] Livingstone and Wagner proved the following theorem.

Theorem 1.1 (Livingstone, Wagner) [7] The number of orbits of $G$ on $k$-subsets of $\Omega$ is less than or equal to the number of orbits on $(k+1)$-subsets.

Alternative proofs were subsequently given by Robinson [8] and Cameron [1] who extended the result to $\Omega$ infinite. An investigation of the cases when equality occurs for $\Omega$ infinite was then made by Cameron [2], [3] and Cameron and Thomas [5]. The case of equality also follows from a stronger "interchange property" examined by Cameron, Neumann and Saxl [4]. In this article, we will prove some similar results about the case of equality when $\Omega$ is finite.

D. Bundy ( $\varangle)$

Mathematisches Seminar, Universität zu Kiel, Ludewig-Meyn Straße 4, Kiel 24098, Germany e-mail: bundy@math.uni-kiel.de

S. Hart

School of Economics, Mathematics and Statistics, Birkbeck, University of London, Malet Street, London WC1E 7HX, UK 
In Section 2 we consider the case when $G$ is intransitive. We show (see Lemma 2.1) that $G$ must have one orbit of length at least $n-k$ and (see Proposition 2.2) that the action of $G$ on this orbit satisfies a strong condition which in almost all cases forces $G$ to be $k$-homogeneous on this orbit.

Transitive but imprimitive groups are then investigated in Section 3. In this case there are too many examples for a complete classification to be feasible, so we concentrate on finding a necessary condition for the sizes and number of blocks in a system of imprimitivity. This quickly reduces to a combinatorial problem of determining when the number of partitions of $k$ into at most $r$ parts of size at most $s$ is the same as for $k+1$. This problem is also of independent interest in invariant theory, where such partitions can be used to count the number of linearly independent semi-invariants of degree $r$ and weight $k$ of a binary form of degree $s$. We are able to determine all the cases of equality for $r \leq 4$ (see Theorem 3.1) and conjecture that for $s \geq r \geq 5$, there are only finitely many cases of equality (see Conjecture 3.2 for details). Theorem 3.7 shows that for $s \geq r \geq 5$, equality can only occur when $2 k \geq r(s-1)-1$, that is $k$ is close to half $n$. We have strong experimental evidence for believing Conjecture 3.2 to be true. We observe that for large enough fixed $r$ and $s$ the number of partitions of $k$ into at most $r$ parts of size at most $s$ approximates to a Gaussian distribution whose peak becomes sharper for larger $r$ and $s$.

In the final section we make some observations about the case when $G$ is primitive. Aside from $(k+1)$-homogeneous groups the only examples we know are the affine general linear groups over a field of size 2 (see Proposition 4.2) and a list of 19 further examples of degree at most 24 , many of which are subgroups of $M_{24}$. The absence in [4] of any examples of degree greater than 24 suggests that such examples may also be rare or non-existent in our situation.

\section{Notation and preliminary results}

For each $0 \leq l \leq n$, let $\sigma_{l}(G)$ be the number of orbits of $G$ on the set of $l$-subsets of $\Omega$. A permutation group is said to be $l$-homogeneous if it is transitive in its action on $l$-subsets, that is $\sigma_{l}(G)=1$. Let $\Delta$ be a $G$-invariant subset of $\Omega$. Then $G^{\Delta}$ will denote the permutation group induced by $G$ in its action on $\Delta$.

Let $H$ be a subgroup of a group $G, \chi$ be a character of $G$ and $\psi$ a character of $H$. Then $\chi \downarrow H$ will denote the restriction of $\chi$ to $H$ and $\psi \uparrow G$ will denote the character induced by $\psi$ on $G$. Furthermore $1_{G}$ will denote the trivial character on $G$.

Lemma 1.2 Let $G \leq \operatorname{Sym}(n), 0 \leq l \leq n$ and $\psi_{l}$ be the character of $\operatorname{Sym}(n)$ induced by the trivial character on $\operatorname{Sym}(l) \times \operatorname{Sym}(n-l)$. Then $\left\langle\psi_{l} \downarrow G, 1_{G}\right\rangle$ is the number of orbits of $G$ on $l$-subsets of $\{1, \ldots, n\}$ and if $0 \leq l<(n-1) / 2$, then $\psi_{l+1}-\psi_{l}$ is an irreducible character of $\operatorname{Sym}(n)$.

Proof See [8].

Lemma 1.3 Let $H \leq G \leq \operatorname{Sym}(n)$ and $1 \leq k<(n-1) / 2$. Then $\sigma_{k+1}(G)-\sigma_{k}(G) \leq$ $\sigma_{k+1}(H)-\sigma_{k}(H)$. In particular, if $\sigma_{k+1}(H)=\sigma_{k}(H)$, then $\sigma_{k+1}(G)=\sigma_{k}(G)$. 
Proof Let $\chi:=\psi_{k+1}-\psi_{k}$ be the irreducible character in the conclusion of Lemma 1.2. Then

$$
\sigma_{k+1}(G)-\sigma_{k}(G)=\left\langle\chi \downarrow G, 1_{G}\right\rangle \leq\left\langle\chi \downarrow H, 1_{H}\right\rangle=\sigma_{k+1}(H)-\sigma_{k}(H) .
$$

In particular, if $\sigma_{k+1}(H)=\sigma_{k}(H)$, then the right-hand side is zero and by Theorem 1.1 the left-hand side is non-negative, so must also be zero.

\section{Intransitive groups with equality}

In this section we investigate intransitive permutation groups which achieve equality in the Livingstone-Wagner Theorem.

Lemma 2.1 Let $G \leq \operatorname{Sym}(n)$ and suppose $\sigma_{k}(G)=\sigma_{k+1}(G)$ for some $1 \leq k<$ $(n-1) / 2$. Then $G$ has an orbit of length at least $n-k$.

Proof Suppose $G$ has no orbit of length at least $n-k$. If $G \leq \operatorname{Sym}(n-l) \times \operatorname{Sym}(l)=$ : $M$, for some $k<l<n-k$, then $\sigma_{k+1}(M)=k+2>k+1=\sigma_{k}(M)$, which contradicts Lemma 1.3. So the sum of the lengths of any set of orbits of $G$ is either at most $k$ or at least $n-k$. In particular, each orbit of $G$ has length at most $k$ and $G$ has at least three orbits.

We claim that there exists a subgroup $M$ of $\operatorname{Sym}(n)$ containing $G$ isomorphic to $\operatorname{Sym}\left(l_{1}\right) \times \operatorname{Sym}\left(l_{2}\right) \times \operatorname{Sym}\left(l_{3}\right)$, where $n=l_{1}+l_{2}+l_{3}$ and $l_{i} \leq k$ for each $1 \leq i \leq 3$. Let $g_{1}$ and $g_{2}$ be the lengths of two distinct orbits of $G$. If $g_{1}+g_{2} \geq n-k$, then the total length of the remaining orbits is at most $k$. Then there exists $M$ isomorphic to $\operatorname{Sym}\left(g_{1}\right) \times \operatorname{Sym}\left(g_{2}\right) \times \operatorname{Sym}\left(n-g_{1}-g_{2}\right)$, which fulfils the claim. Otherwise $g_{1}+g_{2} \leq$ $k$ and there exists a group $G^{\prime}$ containing $G$ which operates as $\operatorname{Sym}\left(g_{1}+g_{2}\right)$ on the union of these two orbits and operates in the same way as $G$ on the other orbits. We then replace $G$ by $G^{\prime}$ and repeat the argument to find $M$. We may assume without loss that $l_{1} \geq l_{2} \geq l_{3}$. It remains to show that $\sigma_{k}(M)<\sigma_{k+1}(M)$, which yields a contradiction to Lemma 1.3.

Note that $\sigma_{k}(M)$ is the number of tuples $\left(a_{1}, a_{2}, a_{3}\right)$ such that $k=a_{1}+a_{2}+a_{3}$ and $a_{i} \leq l_{i}$, for each $1 \leq i \leq 3$. For each such tuple, $\left(a_{1}+1, a_{2}, a_{3}\right)$ corresponds to a suitable partition of $k+1$, except in those cases when $a_{1}=l_{1}$. The number of such exceptional tuples is $k-l_{1}+1$, because $l_{1}+l_{3} \geq n-k>k$ implies that $k-l_{1}<l_{3}$. The tuples of the form $\left(0, a_{2}, a_{3}\right)$, where $a_{2}+a_{3}=k+1$ do not correspond to any partition of $k$ as above. The number of such tuples is $l_{2}+l_{3}-k$, because $a_{2}$ can range between $k+1-l_{3}$ and $l_{2}$. Since $\left(l_{2}+l_{3}-k\right)-\left(k-l_{1}+1\right)=n-(2 k+1)>0$, this shows that $\sigma_{k+1}(M)>\sigma_{k}(M)$ as required.

Proposition 2.2 Let $G \leq \operatorname{Sym}(n)$ and $1 \leq k<(n-1) / 2$ with $\sigma_{k}(G)=\sigma_{k+1}(G)$. Let $\Delta$ be an orbit of $G$ of length at least $n-k$. Then $\sigma_{l}\left(G^{\Delta}\right)=\sigma_{l+1}\left(G^{\Delta}\right)$, for all $k-(n-|\Delta|) \leq l \leq \min (k,|\Delta|-k-2)$.

Proof Note that an orbit of length at least $n-k$ exists by Lemma 2.1. Let $M:=$ $G^{\Delta} \times \operatorname{Sym}(\Omega \backslash \Delta) \geq G$ and let $m:=|\Delta|$. For $t \in \mathbb{N}$, two $t$-subsets of $\Omega$ are in the 
same $M$-orbit if and only if their intersections with $\Delta$ are in the same $G^{\Delta}$-orbit. In particular, these intersections must be of the same size. Hence

$$
\sigma_{t}(M)=\sum_{l=\max (0, t-(n-m))}^{\min (t, m)} \sigma_{l}\left(G^{\Delta}\right) .
$$

Now $m \geq n-k \geq(2 k+1)-k=k+1$. Also $k-(n-m) \geq k+(n-k)-n=0$. Therefore

$$
\begin{aligned}
0 & =\sigma_{k+1}(M)-\sigma_{k}(M)=\sum_{l=k+1-(n-m)}^{k+1} \sigma_{l}\left(G^{\Delta}\right)-\sum_{l=k-(n-m)}^{k} \sigma_{l}\left(G^{\Delta}\right) \\
& =\sigma_{k+1}\left(G^{\Delta}\right)-\sigma_{k-(n-m)}\left(G^{\Delta}\right) .
\end{aligned}
$$

That is, $\sigma_{k+1}\left(G^{\Delta}\right)=\sigma_{k-(n-m)}\left(G^{\Delta}\right)$. If $2 k<m-1$ then the Livingstone-Wagner Theorem forces $\sigma_{l}\left(G^{\Delta}\right)=\sigma_{l+1}\left(G^{\Delta}\right)$, for each $k-(n-m) \leq l \leq k$.

On the other hand, suppose $2 k \geq m-1$. Then $\sigma_{k+1}\left(G^{\bar{\Delta}}\right)=\sigma_{m-(k+1)}\left(G^{\Delta}\right)$ and $m-(k+1)$ is within the range to which the Livingstone-Wagner Theorem applies. We also have that

$$
(m-(k+1))-(k-(n-m))=(n-1)-2 k>0 .
$$

Hence, by the Livingstone-Wagner Theorem, $\sigma_{l}\left(G^{\Delta}\right)=\sigma_{l+1}\left(G^{\Delta}\right)$, for each $k-(n-$ $m) \leq l \leq m-k-2$. Note that $\min (k, m-k-2)$ is $k$ precisely when $2 k<m-1$ and $m-k-2$ otherwise, so the proof is complete.

Proposition 2.2 provides the means to reduce the case of equality for an intransitive group to that of equality for a transitive group. Indeed if $G$ is intransitive with an orbit $\Delta$ satisfying the condition of Proposition 2.2 , then we nearly always have equality $\sigma_{l}\left(G^{\Delta}\right)=\sigma_{l+1}\left(G^{\Delta}\right)$ for several consecutive values of $l$. (If there is just one value of $l$ then either $G$ is already transitive or $n=2 k+2$.) This almost forces $G^{\Delta}$ to be $k$ homogeneous. The only known exceptions with $k<(n-1) / 2$ are where $G^{\Delta} \cong M_{24}$ or $M_{23}$.

\section{Imprimitive groups with equality}

There is an abundance of imprimitive groups which achieve equality in the Livingstone-Wagner Theorem and a complete classification of them seems intractable. Nevertheless, we are able to give a condition on the block sizes which is necessary if equality in the Livingstone-Wagner Theorem holds. Observe that by Lemma 1.3, if $\sigma_{k}(H)=\sigma_{k+1}(H)$ holds for an imprimitive group $H$ with $r$ blocks of size $s$, then $\sigma_{k}(G)=\sigma_{k+1}(G)$, where $G \cong \operatorname{Sym}(s)$ 2 $\operatorname{Sym}(r)$ is the full stabiliser in $\operatorname{Sym}(r s)$ of the blocks of $H$. Note also that the number of orbits of $G$ on $k$-subsets is equal to the number of ways, $P(r, s, k)$, to partition $k$ into at most $r$ parts of size at most $s$. We require $P(r, s, k)=P(r, s, k+1)$. The following result is established by Lemma 3.5, Proposition 3.6 and Proposition 3.9. 
Theorem 3.1 Let $r \in\{2,3,4\}$ with $r \leq s$ and $1 \leq k<(r s-1) / 2$. Then $P(r, s, k)=$ $P(r, s, k+1)$ if and only if one of the following holds.

(a) $r=2$ and $k$ is even.

(b) $r=3$ and

$$
k= \begin{cases}\frac{3 s-3}{2}, & \text { if } s \text { is odd, } \\ \frac{3 s-4}{2}, & \text { if } s \equiv 0 \bmod 4, \\ \frac{3 s-2}{2} \text { or } \frac{3 s-6}{2}, & \text { if } s \equiv 2 \bmod 4 .\end{cases}
$$

(c) $r=4$ and $k=2 s-2$ or $r=s=k=4$.

We also make the following conjecture.

Conjecture 3.2 Let $1<r \leq s, 1 \leq k<(r s-1) / 2$ and suppose $P(r, s, k)=$ $P(r, s, k+1)$. Then one of the following holds:

(a) $r \in\{2,3,4\}$ and the possibilities for $s$ and $k$ are as in Theorem 3.1; or

(b) $r, s$ and $k$ have the values given by a column of the following table

\begin{tabular}{c|ccccccccc}
$r$ & 5 & 5 & 5 & 6 & 6 & 6 & 6 & 6 & 7 \\
\hline$s$ & 6 & 10 & 14 & 6 & 7 & 9 & 11 & 13 & 10 \\
\hline$k$ & 14 & 24 & 34 & 16 & 20 & 26 & 32 & 38 & 34
\end{tabular}

Remark 3.3 The quantity $P(r, s, k)-P(r, s, k-1)$ is of interest in invariant theory. By a theorem of Cayley and Sylvester (see Satz 2.21 of [9]) it is equal to the number of linearly independent semi-invariants of degree $r$ and weight $k$ of a binary form of degree $s$. Conjecture 3.2, if proven, would then give the values of $r, s$ and $k$ for which no such semi-invariant exists.

We now define some more notation which we will use in this section. Let $\mathcal{P}(r, s, k)$ be the set of partitions of $k$ into at most $r$ parts of size at most $s$, so $P(r, s, k)=$ $|\mathcal{P}(r, s, k)|$. We will use the convention that $P(r, s, k)=0$ if $k<0$ or $k>r s$. By considering dual partitions we observe that $P(r, s, k)=P(s, r, k)$, so without loss we will assume that $r \leq s$. Elements of $\mathcal{P}(r, s, k)$ will be written $\left(a_{1}, a_{2}, \ldots, a_{r}\right)$ where $\sum_{i=1}^{r} a_{i}=k$ and $s \geq a_{1} \geq \cdots \geq a_{r} \geq 0$. Let $\mathcal{A}(r, s, k)$ be the subset of $\mathcal{P}(r, s, k)$ consisting of all partitions of the form $\left(s, a_{2}, \ldots, a_{r}\right)$ and let $\mathcal{B}(r, s, k+1)$ be the subset of $\mathcal{P}(r, s, k+1)$ consisting of all partitions of the form $\left(x, x, a_{3}, \ldots, a_{r}\right)$, for some $x \leq s$. Furthermore, let $A(r, s, k)=|\mathcal{A}(r, s, k)|$ and $B(r, s, k)=|\mathcal{B}(r, s, k)|$. Note that $A(r, s, k)=P(r-1, s, k-s)$. We will define a bijection from a subset of $\mathcal{P}(r, s, k)$ to a subset of $\mathcal{P}(r, s, k+1)$. Let $\left(a_{1}, a_{2}, \ldots, a_{r}\right) \in \mathcal{P}(r, s, k)$ with $s>a_{1} \geq$ $a_{2} \geq \ldots \geq a_{r} \geq 0$, and define

$$
f\left(a_{1}, a_{2}, \ldots, a_{r}\right)=\left(a_{1}+1, a_{2}, \ldots, a_{r}\right) .
$$

Then $f$ is a bijection from $\mathcal{P}(r, s, k) \backslash \mathcal{A}(r, s, k)$ to $\mathcal{P}(r, s, k+1) \backslash \mathcal{B}(r, s, k+1)$. In particular we have the following result.

Lemma 3.4 Let $r, s, k \geq 1$. Then

$$
P(r, s, k+1)-P(r, s, k)=B(r, s, k+1)-A(r, s, k) .
$$


So the problem of determining when $P(r, s, k)=P(r, s, k+1)$ reduces to that of determining when $B(r, s, k+1)=A(r, s, k)$. We now consider in turn the cases when $r=2,3$ and 4 .

Lemma 3.5 Let $s \geq 0$. Then

$$
P(2, s, k)= \begin{cases}0, & \text { if } k>2 s, \text { or } k<0, \\ s-\left\lceil\frac{k}{2}\right\rceil+1, & \text { if } s \leq k \leq 2 s, \\ \left\lfloor\frac{k}{2}\right\rfloor+1, & \text { if } 0 \leq k \leq s .\end{cases}
$$

In particular, if $1 \leq k<s$, then $P(2, s, k)=P(2, s, k+1)$ if and only if $k$ is even.

Proof Elementary.

Proposition 3.6 Let $s \geq 3$ and $1 \leq k<(3 s-1) / 2$. Then $P(3, s, k)=P(3, s, k+1)$ if and only if one of the following holds:

(a) $s$ is odd and $k=(3 s-3) / 2$,

(b) $s \equiv 0 \bmod 4$ and $k=(3 s-4) / 2$,

(c) $s \equiv 2 \bmod 4$ and $k=(3 s-2) / 2$ or $(3 s-6) / 2$.

Proof Let $d_{k}=P(3, s, k+1)-P(3, s, k)=B(3, s, k+1)-A(3, s, k)$. By Lemma 3.5,

$$
A(3, s, k)=P(2, s, k-s)= \begin{cases}\left\lfloor\frac{k-s}{2}\right\rfloor+1 & \text { if } s \leq k<(3 s-1) / 2 \\ 0 & \text { if } k<s\end{cases}
$$

Moreover,

$$
B(3, s, k+1)=|\{(a, a, b) \mid s \geq a \geq b, 2 a+b=k+1\}|=\left\lfloor\frac{k+1}{2}\right\rfloor-\left\lceil\frac{k+1}{3}\right\rceil+1 .
$$

Hence

$$
B(3, s, k+1) \geq \frac{k}{2}-\frac{k+3}{3}+1=\frac{k}{6}>0 .
$$

So if $A(3, s, k)=0$, then $d_{k} \geq k / 6>0$. We may therefore assume that

$$
s \leq k<(3 s-1) / 2 \text { and } A(3, s, k)=\left\lfloor\frac{k-s}{2}\right\rfloor+1 .
$$

Thus

$$
d_{k}=\left\lfloor\frac{k+1}{2}\right\rfloor-\left\lceil\frac{k+1}{3}\right\rceil-\left\lfloor\frac{k-s}{2}\right\rfloor .
$$

Suppose $s$ is odd. Then $k+1 \equiv k-s \bmod 2$. Hence

$$
d_{k}=\frac{k+1-(k-s)}{2}-\left\lceil\frac{k+1}{3}\right\rceil=\frac{s+1}{2}-\left\lceil\frac{k+1}{3}\right\rceil \text {. }
$$


Therefore

$$
d_{k}=0 \Leftrightarrow k \in\left\{\frac{3 s+1}{2}, \frac{3 s-1}{2}, \frac{3 s-3}{2}\right\} .
$$

Since $k<(3 s-1) / 2$, this forces $k=\frac{3 s-3}{2}$.

Suppose $s$ is even. Then

$$
d_{k} \geq \frac{k}{2}-\frac{k+3}{3}-\frac{k-s}{2}=\frac{1}{6}(3 s-2 k-6) .
$$

Assume $d_{k}=0$. Then $2 k \geq 3 s-6$. Thus $3 s / 2-3 \leq k \leq 3 s / 2-1$ and so $\left\lceil\frac{k+1}{3}\right\rceil=\frac{s}{2}$. Therefore

$$
d_{k}= \begin{cases}\frac{k}{2}-\frac{s}{2}-\frac{k-s}{2}=0, & \text { if } k \text { is even } \\ \frac{k+1}{2}-\frac{s}{2}-\frac{k-s-1}{2}=1, & \text { if } k \text { is odd, a contradiction. }\end{cases}
$$

Thus $k$ is even, $\frac{3 s-6}{2} \leq k \leq \frac{3 s-2}{2}$ and hence

$$
k=\left\{\begin{array}{lll}
\frac{3 s-4}{2} & \text { if } s \equiv 0 \quad \bmod 4 \\
\frac{3 s-2}{2} \text { or } \frac{3 s-6}{2} & \text { if } s \equiv 2 \quad \bmod 4 .
\end{array}\right.
$$

Theorem 3.7 Let $4 \leq r \leq s$ and $1 \leq k<(r s-1) / 2$. If $P(r, s, k)=P(r, s, k+1)$, then $k \geq(r(s-1)-1) / 2$ or $r=s=k=4$.

Proof Suppose first that $k<s$. Then $A(r, s, k)=0$ but $B(r, s, k)>0$, since $r \geq 4$. Therefore by Lemma $3.4 P(r, s, k)<P(r, s, k+1)$. Now suppose that $k=s \geq 5$. Then

$$
P(r, s, k)=P(r, k, k)=2+P(r, k-2, k)
$$

and

$$
P(r, s, k+1)=P(r, k, k+1)=3+P(r, k-2, k+1) .
$$

Since $(r(k-2)-1) / 2 \geq(4(k-2)-1) / 2=2 k-9 / 2>k$, applying Theorem 1.1 yields $P(r, k-2, k) \leq P(r, k-2, k+1)$ and so $P(r, s, k)<P(r, s, k+1)$ in this case.

It remains to show for $s<k<(r(s-1)-1) / 2$ that $P(r, s, k)<P(r, s, k+1)$. So we assume for a contradiction that $P(r, s, k)=P(r, s, k+1)$ in this case. Observe that

$$
P(r, s, k)=P(r, s-1, k)+P(r-1, s, k-s) .
$$

Since $k<(r(s-1)-1) / 2$ and $k-s<(r(s-1)-1-2 s) / 2<((r-1) s-1) / 2$, by Theorem 1.1, $P(r, s-1, k) \leq P(r, s-1, k+1)$ and $P(r-1, s, k-s) \leq P(r-1$, $s, k-s+1)$. So under our assumption we have $P(r-1, s, k-s)=P(r-1, s$, $k-s+1)$. We now proceed by induction on $r$. 
Suppose first that $r=4$. Then by Proposition 3.6, $P(3, s, k-s)=P(3, s, k-$ $s+1)$ implies $3 s / 2-3 \leq k-s \leq 3 s / 2-1$. However $k<(4(s-1)-1) / 2=2 s-5 / 2$, so $k-s \leq s-3<3 s / 2-3$, a contradiction.

Now suppose $r>4$ and the result holds for $r-1$ in place of $r$. Since $P(r-1, s$, $k-s)=P(r-1, s, k-s+1)$, we obtain by induction that

$$
k-s \geq \frac{(r-1)(s-1)-1}{2}=\frac{r s-r-s}{2} .
$$

Hence $k \geq(r s-r+s) / 2>(r s-1) / 2$, a contradiction. Therefore by induction the result holds for all $r \geq 4$.

Proposition 3.8 Let $s \geq 4$ and $2 s-2 \leq k \leq 2 s-1$. Then $P(4, s, k)=P(4, s, k+1)$ if and only if $k=2 s-2$.

Proof Since $r=4$ is fixed, for this proof we will abbreviate $A(r, s, k)$ by $A(s, k)$ and $B(r, s, k)$ by $B(s, k)$. We first show that for all $s \geq 4, P(4, s, 2 s-2)=$ $P(4, s, 2 s-1)$. We need to evaluate $B(s, k)$ more precisely. Now

$$
\mathcal{B}(s, k)=\{(a, a, b, c): s \geq a \geq b \geq c \geq 0,2 a+b+c=k\} .
$$

Now $0 \leq b+c \leq 2 a$ implies $2 a \leq k \leq 4 a$. Hence $\left\lceil\frac{k}{4}\right\rceil \leq a \leq\left\lfloor\frac{k}{2}\right\rfloor$. Thus

$$
B(s, k)=\sum_{a=\left\lceil\frac{k}{4}\right\rceil}^{\left\lfloor\frac{k}{2}\right\rfloor} P(2, a, k-2 a) .
$$

By Lemma 3.5, the value of $P(2, a, k-2 a)$ depends on whether $0 \leq k-2 a \leq a$ or $a \leq k-2 a \leq 2 a$. Now $2 a-(k-2 a)=4 a-k \geq 0$. Also $k-2 a \geq a$ whenever $a \leq\left\lfloor\frac{k}{3}\right\rfloor$. Therefore by Lemma 3.5

$$
B(s, k)=\sum_{a=\left\lceil\frac{k}{4}\right\rceil}^{\left\lfloor\frac{k}{3}\right\rfloor}\left(a-\left\lceil\frac{k-2 a}{2}\right\rceil+1\right)+\sum_{a=\left\lfloor\frac{k}{3}\right\rfloor+1}^{\left\lfloor\frac{k}{2}\right\rfloor}\left(\left\lfloor\frac{k-2 a}{2}\right\rfloor+1\right) .
$$

It follows that

$$
\begin{aligned}
B(s, 2 s-1)= & \sum_{a=\left\lceil\frac{2 s-1}{4}\right\rceil}^{\left\lfloor\frac{2 s-1}{3}\right\rfloor}\left(a-\left\lceil\frac{2 s-1-2 a}{2}\right\rceil+1\right)+\sum_{a=\left\lfloor\frac{2 s-1}{3}\right\rfloor+1}^{\left\lfloor\frac{2 s-1}{2}\right\rfloor}\left(\left\lfloor\frac{2 s-1-2 a}{2}\right\rfloor+1\right) \\
= & \sum_{a=\left\lceil\frac{s}{2}\right\rceil}^{\left\lfloor\frac{2 s-1}{3}\right\rfloor}(2 a-s+1)+\sum_{a=\left\lfloor\frac{2 s-1}{3}\right\rfloor+1}^{s-1}(s-a) \\
= & (1-s)\left(\left\lfloor\frac{2 s-1}{3}\right\rfloor-\left\lceil\frac{s}{2}\right\rceil+1\right)+\left\lfloor\frac{2 s-1}{3}\right\rfloor\left(\left\lfloor\frac{2 s-1}{3}\right\rfloor+1\right)-\left\lceil\frac{s}{2}\right\rceil\left(\left\lceil\frac{s}{2}\right\rceil-1\right) \\
& +s\left(s-1-\left\lfloor\frac{2 s-1}{3}\right\rfloor\right)-\frac{1}{2}(s-1) s+\frac{1}{2}\left\lfloor\frac{2 s-1}{3}\right\rfloor\left(\left\lfloor\frac{2 s-1}{3}\right\rfloor+1\right)
\end{aligned}
$$




$$
\begin{aligned}
= & \left\lfloor\frac{2 s-1}{3}\right\rfloor\left(\frac{3}{2}\left\lfloor\frac{2 s-1}{3}\right\rfloor+1+1-s-s+\frac{1}{2}\right)+\left\lceil\frac{s}{2}\right\rceil\left(-\left\lceil\frac{s}{2}\right\rceil+s-1+1\right) \\
& +1-s+s^{2}-s-\frac{1}{2} s^{2}+\frac{1}{2} s
\end{aligned}
$$

$B(s, 2 s-1)=\underbrace{\frac{1}{2}\left\lfloor\frac{2 s-1}{3}\right\rfloor\left(3\left\lfloor\frac{2 s-1}{3}\right\rfloor+5-4 s\right)}_{X_{3}(B)}+\underbrace{\left\lceil\frac{s}{2}\right\rceil\left(s-\left\lceil\frac{s}{2}\right\rceil\right)+\frac{1}{2}\left(s^{2}-3 s+2\right)}_{X_{2}(B)}$

We now work out $A(s, 2 s-2)$ in a similar fashion. Firstly note that $A(s, 2 s-2)=$ $P(3, s, s-2)=P(3, s-2, s-2)$, and

$$
P(3, s-2, s-2)=\#\{a, b, c: a \geq b \geq c \geq 0, a+b+c=s-2\} .
$$

This implies that $\left\lceil\frac{s-2}{3}\right\rceil \leq a \leq s-2$. Thus $A(s, 2 s-2)=\sum_{a=\left\lceil\frac{s-2}{3}\right\rceil}^{s-2} P(2, a$, $s-2-a)$. From Lemma 3.5, and noting that $s-2-a \geq a$ when $a \leq\left\lfloor\frac{s-2}{2}\right\rfloor$, we make the following calculation.

$$
\begin{aligned}
A(s, 2 s-2)= & \sum_{a=\left\lceil\frac{s-2}{3}\right\rceil}^{\left\lfloor\frac{s-2}{2}\right\rfloor}\left(a-\left\lceil\frac{s-2-a}{2}\right\rceil+1\right)+\sum_{a=\left\lfloor\frac{s-2}{2}\right\rfloor+1}^{s-2}\left(\left\lfloor\frac{s-2-a}{2}\right\rfloor+1\right) \\
= & \sum_{a=\left\lceil\frac{s-2}{3}\right\rceil}^{\left\lfloor\frac{s}{2}\right\rfloor-1}(a-(s-2-a))+\sum_{a=\left\lceil\frac{s-2}{3}\right\rceil}^{s-2}\left(\left\lfloor\frac{s-a}{2}\right\rfloor\right) \\
= & \sum_{a=\left\lceil\frac{s-2}{3}\right\rceil}^{\left\lfloor\frac{s}{2}\right\rfloor-1}(2 a-s+2) \\
& +\sum_{a=\left\lceil\frac{s-2}{3}\right\rceil}^{s-2}\left(\frac{s-a}{2}\right)-\frac{1}{2} \#\left\{i \in\left\{2, \ldots, s-\left\lceil\frac{s-2}{3}\right\rceil\right\}: i \text { odd }\right\}
\end{aligned}
$$

Now the number of odd numbers in the range $\{2, \ldots, x\}$ is $\left\lfloor\frac{x-1}{2}\right\rfloor$, so the number of odd numbers in $\left\{2, \ldots, s-\left\lceil\frac{s-2}{3}\right\rceil\right\}$ is $\left\lfloor\frac{\left\lfloor\frac{2 s+2}{3}\right\rfloor-1}{2}\right\rfloor=\left\lfloor\frac{2 s-1}{6}\right\rfloor=\left\lfloor\frac{s-1}{3}\right\rfloor$. Therefore

$$
\begin{aligned}
A(s, 2 s-2)= & (2-s)\left(\left\lfloor\frac{s}{2}\right\rfloor-\left\lceil\frac{s-2}{3}\right\rceil\right)+\left\lfloor\frac{s}{2}\right\rfloor\left(\left\lfloor\frac{s}{2}\right\rfloor-1\right)-\left\lceil\frac{s-2}{3}\right\rceil\left(\left\lceil\frac{s-2}{3}\right\rceil-1\right) \\
& +\frac{s}{2}\left(s-2-\left\lceil\frac{s-2}{3}\right\rceil+1\right)-\frac{1}{4}(s-2)(s-1) \\
& +\frac{1}{4}\left\lceil\frac{s-2}{3}\right\rceil\left(\left\lceil\frac{s-2}{3}\right\rceil-1\right)-\frac{1}{2}\left\lfloor\frac{s-1}{3}\right\rfloor \\
= & \left\lfloor\frac{s}{2}\right\rfloor\left(2-s+\left\lfloor\frac{s}{2}\right\rfloor-1\right)+\frac{s}{2}(s-1)-\frac{1}{4}(s-2)(s-1) \\
& +\left\lceil\frac{s-2}{3}\right\rceil\left(s-2-\frac{3}{4}\left\lceil\frac{s-2}{3}\right\rceil+\frac{3}{4}-\frac{s}{2}\right)-\frac{1}{2}\left\lfloor\frac{s-1}{3}\right\rfloor
\end{aligned}
$$




$$
\begin{aligned}
A(s, 2 s-2)= & \underbrace{\left\lfloor\frac{s}{2}\right\rfloor\left(\left\lfloor\frac{s}{2}\right\rfloor+1-s\right)+\frac{1}{4}(s-1)(s+2)}_{X_{2}(A)} \\
& +\underbrace{\frac{1}{4}\left\lceil\frac{s-2}{3}\right\rceil\left(2 s-5-3\left\lceil\frac{s-2}{3}\right\rceil\right)-\frac{1}{2}\left\lfloor\frac{s-1}{3}\right\rfloor}_{X_{3}(A)} .
\end{aligned}
$$

Now $P(4, s, 2 s-2)=P(4, s, 2 s-1)$ if and only if $B(s, 2 s-1)=A(s, 2 s-2)$, which is if and only if $X_{2}(B)-X_{2}(A)=X_{3}(A)-X_{3}(B)$. We have

$$
\begin{gathered}
X_{2}(B)-X_{2}(A)=\left(\left\lceil\frac{s}{2}\right\rceil\left(s-\left\lceil\frac{s}{2}\right\rceil\right)+\frac{1}{2}\left(s^{2}-3 s+2\right)\right) \\
-\left(\left\lfloor\frac{s}{2}\right\rfloor\left(\left\lfloor\frac{s}{2}\right\rfloor+1-s\right)+\frac{1}{4}(s-1)(s+2)\right) .
\end{gathered}
$$

A simple calculation shows that regardless of whether $s$ is odd or even, $X_{2}(B)-$ $X_{2}(A)=\frac{1}{4}\left(3 s^{2}-9 s+6\right)$.

$$
\begin{aligned}
& X_{3}(A)-X_{3}(B)=\left(\frac{1}{4}\left\lceil\frac{s-2}{3}\right\rceil\left(2 s-5-3\left\lceil\frac{s-2}{3}\right\rceil\right)-\frac{1}{2}\left\lfloor\frac{s-1}{3}\right\rfloor\right) \\
& -\left(\frac{1}{2}\left\lfloor\frac{2 s-1}{3}\right\rfloor\left(3\left\lfloor\frac{2 s-1}{3}\right\rfloor+5-4 s\right)\right) .
\end{aligned}
$$

Calculating for each possible value of $s$ modulo 3 shows that in each case, $X_{3}(A)-X_{3}(B)=\frac{1}{4}\left(3 s^{2}-9 s+6\right)=X_{2}(B)-X_{2}(A)$. Therefore, for all $s \geq 4$, $P(4, s, 2 s-2)=P(4, s, 2 s-1)$.

We now show that $P(4, s, 2 s-1)<P(4, s, 2 s)$ for all $s \geq 4$. Since $P(4, s$, $2 s-2)=P(4, s, 2 s-1)$ for all $s \geq 4$, by substituting $s+1$ for $s$ in Lemma 3.4 we have

$$
A(s+1,2 s)=B(s+1,2 s+1) .
$$

Now $A(s, 2 s-1)=P(3, s, s-1)=P(3, s-1, s-1)$ as no part of a partition of $s-1$ can exceed $s-1$. Similarly $A(s+1,2 s)=P(3, s+1, s-1)=P(3, s-1, s-1)$. Hence

$$
A(s, 2 s-1)=A(s+1,2 s) .
$$

Now we consider $B(s+1,2 s+1)$ compared to $B(s, 2 s)$.

Setting $k+1=2 s$ and $k+1=2 s+1$ in (1), respectively, gives:

$$
\begin{aligned}
B(s, 2 s) & =\sum_{a=\left\lceil\frac{s}{2}\right\rceil}^{\left\lfloor\frac{2 s}{3}\right\rfloor}(a-(s-a)+1)+\sum_{a=\left\lfloor\frac{2 s}{3}\right\rfloor+1}^{s}(s-a+1) \\
& =\sum_{a=\left\lceil\frac{s}{2}\right\rceil}^{\left\lfloor\frac{2 s}{3}\right\rfloor}(2 a-s+1)+\sum_{a=\left\lfloor\frac{2 s}{3}\right\rfloor+1}^{s}(s-a+1) ;
\end{aligned}
$$




$$
\begin{aligned}
B(s+1,2 s+1) & =\sum_{a=\left\lceil\frac{2 s+1}{4}\right\rceil}^{\left\lfloor\frac{2 s+1}{3}\right\rfloor}(a-(s-a+1)+1)+\sum_{a=\left\lfloor\frac{2 s+1}{3}\right\rfloor+1}^{s}((s-a)+1) \\
& =\sum_{a=\left\lceil\frac{s+1}{2}\right\rceil}^{\left\lfloor\frac{2 s+1}{3}\right\rfloor}(2 a-s)+\sum_{a=\left\lfloor\frac{2 s+1}{3}\right\rfloor+1}^{s}(s-a+1) .
\end{aligned}
$$

If $\left\lfloor\frac{2 s}{3}\right\rfloor=\left\lfloor\frac{2 s+1}{3}\right\rfloor$, then $B(s, 2 s)-B(s+1,2 s+1) \geq \sum_{a=\lceil s / 2\rceil}^{\lfloor(2 s+1) / 3\rfloor} 1 \geq \frac{2 s-1}{3}-\frac{s-1}{2}>0$. If $\left\lfloor\frac{2 s}{3}\right\rfloor<\left\lfloor\frac{2 s+1}{3}\right\rfloor$ then $\left\lfloor\frac{2 s}{3}\right\rfloor=\frac{2 s-2}{3},\left\lfloor\frac{2 s+1}{3}\right\rfloor=\frac{2 s+1}{3}$ and

$$
\begin{aligned}
B(s, 2 s)-B(s+1,2 s+1) \geq & \left(\sum_{a=\left\lceil\frac{s}{2}\right\rceil}^{\left\lfloor\frac{2 s}{3}\right\rfloor} 1\right)-\left(2\left\lfloor\frac{2 s+1}{3}\right\rfloor-s\right) \\
& +\left(s-\left(\left\lfloor\frac{2 s}{3}\right\rfloor+1\right)+1\right) \\
\geq & \frac{2 s-2}{3}-\frac{s-1}{2}-\frac{4 s+2}{3}+2 s-\frac{2 s-2}{3} \\
= & \frac{1}{6}(-3 s+3-8 s-4+12 s)=\frac{1}{6}(s-1)>0 .
\end{aligned}
$$

Thus in any case $B(s, 2 s)>B(s+1,2 s+1)$. Therefore by equations (2) and (3),

$$
B(s, 2 s)-A(s, 2 s-1)>B(s+1,2 s+1)-A(s+1,2 s)=0 .
$$

Hence by Lemma $3.4 P(4, s, 2 s-1)<P(4, s, 2 s)$.

Proposition 3.9 Let $s \geq 4$ and $1 \leq k \leq 2 s-1$. Then $P(4, s, k)=P(4, s, k+1)$ if and only if $k=2 s-2$ or $s=k=4$.

Proof In the case $s=k=4$ it can be easily computed that $P(4,4,4)=P(4,4,5)=$ 5. Otherwise, by Theorem 3.7, if $P(4, s, k)=P(4, s, k+1)$, then $4(s-1)-1 \leq 2 k$ and, since $k$ is an integer, $2 s-2 \leq k$. We may now apply Proposition 3.8 to get the result.

Theorem 3.1 now follows immediately from Lemma 3.5, Proposition 3.6 and Proposition 3.9.

\section{Primitive groups with equality}

Primitive groups which are not $(k+1)$-homogeneous but achieve equality in the Livingstone-Wagner Theorem for some $k<(n-1) / 2$ are fairly rare. Searching the database of primitive groups in GAP [6] for degrees up to 28 produced the list given below. The lack of any primitive examples in the more special situation in [4] for degrees greater than 24 suggests very tentatively that this may be the complete list. 
It might be possible to prove such a result using the O'Nan-Scott Theorem and the Classification of Finite Simple Groups, but such a proof would certainly be very labourious and we do not attempt this here. The difficulty in finding a more enlightening approach to this problem is that one would like to exploit the high degree of transitivity in the examples below. In particular it would be useful to have a result relating the number of orbits of a group to that of its point-stabilizers, but a simple relationship in general does not appear to exist. We therefore leave this problem open.

Remark 4.1 The known primitive but not $(k+1)$-homogeneous groups $G$ such that $\sigma_{k}(G)=\sigma_{k+1}(G)$, for some $k<(n-1) / 2$, are:

(a) $A G L(m, 2)$, for $m \geq 4, n=2^{m}, k=4$,

(b) $A S L(2,3)$ or $A G L(2,3)$, for $n=9, k=3$,

(c) $\operatorname{Sym}(5), \operatorname{Sym}(6), P G L(2,9)$ or $P \Gamma L(2,9)$, for $n=10, k=4$,

(d) $M_{11}, P S L(2,11), P G L(2,11)$, for $n=12, k=4$,

(e) $\operatorname{PSL}(3,3)$, for $n=13, k=4$,

(f) $P G L(2,13)$, for $n=14, k=4$,

(g) $2^{4}: \operatorname{Alt}(6), 2^{4}: \operatorname{Sym}(6), 2^{4}: \operatorname{Alt}(7)$, for $n=16, k=6$,

(h) $P G L(2,17)$, for $n=18, k=6$ or 8 ,

(i) $M_{22}$ or $\operatorname{Aut}\left(M_{22}\right)$, for $n=22, k=8$,

(j) $M_{23}$, for $n=23, k=8,9$,

(k) $M_{24}$, for $n=24, k=6,8,9$ or 10 .

Observe that many of these groups are subgroups of $M_{24}$.

Regarding case (a), we prove the following.

Proposition 4.2 Let $G=A G L(m, 2)$, for $m \geq 4$, acting naturally on an $m$ dimensional vector space $V$ over $G F(2)$. Then $\sigma_{4}(G)=\sigma_{5}(G)=2$.

Proof Observe that the stabiliser in $G$ of any three points of $V$ fixes the fourth point in the unique affine plane containing these three points and is transitive on the remaining points of $V$. It follows that $\sigma_{4}(G)=2$ and also $G$ has a single orbit on the set of 5subsets which contain affine planes. Let $\Delta$ be any set of five distinct points in $V$ which does not contain any affine plane. Then $\Delta$ is not contained in an affine 3-dimensional subspace of $V$. Furthermore the stabiliser in $G$ of an affine 3-dimensional subspace $W$ is transitive on pairs $(\alpha, \Lambda)$, where $\alpha$ is a point not in $W$ and $\Lambda$ is any set of four points in $W$ which is not an affine plane. Therefore $G$ has a single orbit on 5-subsets which do not contain any affine plane. Thus $\sigma_{5}(G)=2$.

\section{References}

1. Cameron, P.J.: Transitivity of permutation groups on unordered sets. Math. Z. 148(2), 127-139 (1976)

2. Cameron, P.J.: Orbits of permutation groups on unordered sets. J. London Math. Soc. (2) 17(3), 410414 (1978)

3. Cameron, P.J.: Orbits of permutation groups on unordered sets. II. J. London Math. Soc. (2) 23(2), 249-264 (1981) 
4. Cameron, P.J., Neumann, P.M., Saxl, J.: An interchange property in finite permutation groups. Bull. London Math. Soc. 11(2), 161-169 (1979)

5. Cameron, P.J., Thomas, S.: Groups acting on unordered sets. Proc. London Math. Soc. (3) 59(3), 541557 (1989)

6. GAP_-Groups, Algorithms and Programming, Version 4.4.4 (2004). http://www.gap-system.org/

7. Livingstone, D., Wagner, A.: Transitivity of finite permutation groups on unordered sets. Math. Z. 90, 393-403 (1965)

8. Robinson, G. de B.: Note on a theorem of Livingstone and Wagner. Math. Z. 102, 351-352 (1967)

9. Schur, I.: Vorlesungen Über Invariantentheorie. Bearbeitet und herausgegeben von Helmut Grunsky. Die Grundlehren der mathematischen Wissenschaften, Band 143. Springer, Berlin (1968) 